\title{
A few acquisition trials: Effects of magnitude and percent reward
}

\section{AMADO M. PADILLA \\ UNIVERSITY OF NEWMEXICO}

The effects of magnitude and percent reward on resistance to extinction were investigated with four acquisition trials. It was found that differential magnitudes of reward resulted in significantly different running speeds in acquisition and that partially rewarded $S s$ showed greater resistance to extinction than continuously rewarded Ss. The results are discussed in terms of the Amsel-Spence frustration hypothesis.

McCain (1965a, b; 1966) has reported several studies concerned with the partial reinforcement effect (PRE) following a small number of acquisition trials. One study in this series (McCain, 1966, Experiment 3) attempted to partial out the effects of magnitude of reward on extinction performance. In this study, McCain found no difference in either acquisition or extinction between large and small continuously rewarded groups, although both groups were less resistant to extinction than a partially rewarded group. In contrast, amount of reward is known to affect both acquisition and extinction following extensive training. The present study was designed to investigate further the effects of magnitude and percent of reward on resistance to extinction following a few acquisition trials.

Subjects

The Ss were 28 naive female hooded rats approximately 140 days old at the start of training.

Apparatus

The double alley enclosed runway used in this experiment has been described in detail elsewhere (Logan, 1960). For this study, the $4 \mathrm{ft}$. straight black alley was used, terminating at a food cup into which $45 \mathrm{mg}$ Noyes reward pellets were placed by $\mathrm{E}$ prior to the start of rewarded acquisition trials, Running speed, the reciprocal of time between a photobeam 1 in. from the start door and a photobeam 1 in. in front of the goal cup, was the measure used in the analyses.

\section{Procedure}

The Ss were placed on a $23 \mathrm{~h}$ food deprivation schedule on Day 1 with water freely available during the entire experiment. On Days 2-6 all Ss were removed from their individual cages and handled for $1 \mathrm{~h}$ with food available on the handling table during this period. On Days 7-10 Ss were allowed to explore the alley without reward for $15 \mathrm{~min}$ in groups of three. After exploration Ss were returned to the handling table and allowed to eat dry lab chow and $45 \mathrm{mg}$ Noyes pellets for $20 \mathrm{~min}$ and then returned to their home cages.
On Day $11 \mathrm{Ss}$ were given $20 \mathrm{~min}$ of access to Noyes pellets only. Following this Ss were randomly assigned to experimental conditions. There were six Ss in each of four experimental conditions and four Ss in the control group. The experimental groups were designated as: $100 \%$ large reward, $100 \%$ small reward, $50 \%$ large reward, and $50 \%$ small reward. The control group was not rewarded for running in the alley.

On Day 12 each $\mathrm{S}$ was given four acquisition trials with the large reward groups receiving $13,45 \mathrm{mg}$ pellets and the small reward groups recelving $2,45 \mathrm{mg}$ pellets. The $100 \%$ groups were rewarded for running on all acquisition trials. The 50\% groups were rewarded on Trials 2 and 4. Following acquisition Ss were run for 13 extinction trials. The intertrial interval in both acquisition and extinction was approximately $3 \mathrm{~min}$. Any animal requiring 60 or more sec to break the photobeam in front of the goal cup was guided to the goal box and assigned a maximum running speed of .01. The empty food cup was present in the goal box on nonrewarded trials during both acquisition and extinction.

Results

The mean running speed in acquisition and extinction for the five groups is shown in Fig. 1. First trial extinction performance for all groups is shown separately since this trial cannot be considered a true

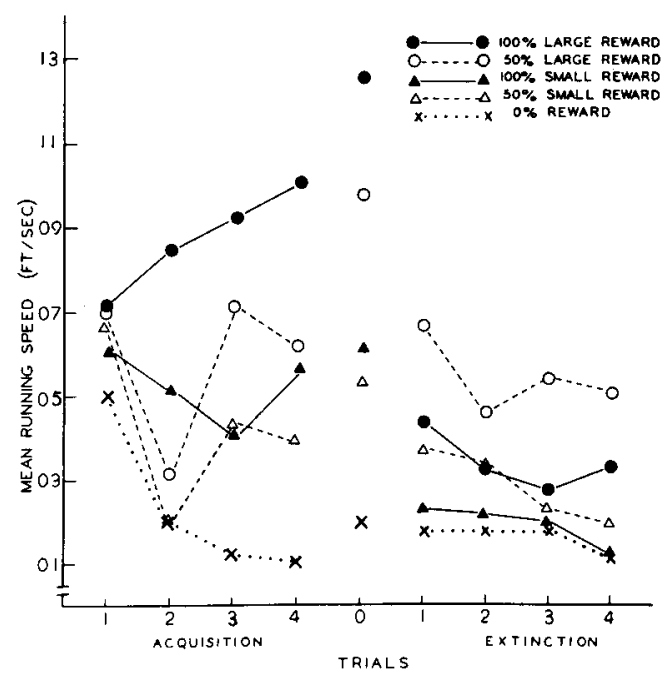

Fig. 1. Mean running speed for acquisition trials and blocks of three extinction trials. 
extinction trial and depicts the asymptotic acquisition performance attained by each of the groups. As can be seen in Fig. 1 , both large reward groups $(100 \%$ and $50 \%$ ) ran faster than the small reward groups. Moreover, all of the experimental groups ran faster than the control animals. Mean running speed for each of the experimental groups over acquisition Trials 3 and 4 and Trial 0 of extinction was tested by a 2 by 2 analysis of variance. The magnitude of reward effect was significant at the .005 level $(F=$ $10.40, \mathrm{df}=1 / 20)$ and neither percent reward $(F=1.12$, $\mathrm{df}=1 / 20, p>.05$ ) effect nor the magnitude of reward by percent reward $(F<1)$ interaction were significant.

As can be seen in Fig. 1, both of the partially rewarded groups demonstrated greater resistance to extinction than their equal magnitude of reward $100 \%$ comparison groups. Since the final acquisition performance levels of the four groups differed markedly, a rate-of-extinction statistic (Anderson, 1963, Eq. 4) was used for each $S$ on each speed score over the 12 trials of extinction. Analysis of variance of these data revealed that the partially rewarded animals were significantly more resistant to extinction than the continuously rewarded Ss $(F=9.88, \mathrm{df}=1 / 20, p<.01)$. Magnitude of reward was not significant $(p>.05)$, nor was the interaction of percent reward by magnitude of reward. Individual comparisons between the various conditions in extinction indicated that the 50\% large reward condition differed significantly from the $100 \%$ large reward group $(t=2.98, \mathrm{df}=10, \mathrm{p}<.01)$ and from the $100 \%$ small reward group $(t=3.01, d f=10, p<.01)$ with all other comparisons failing to achieve significance $(p>.05)$. Moreover, all the experimental groups with the possible exception of the $100 \%$ small reward Ss ran faster in extinction than the control Ss.

\section{Discussion}

The acquisition performance indicated that magnitude of reward differences occurred with just four training trials. This finding is in accord with investigations reporting similar results (e.g., Logan, 1960) after more extensive training. The interesting feature of these data is the rapidity with which differential running speeds were established for the animals in the two amount of reinforcement conditions. As noted earlier, McCain (1966, Experiment 3) found no difference in acquisition or extinction for groups given different amounts of reward.

The greater resistance to extinction of the $50 \%$ reward groups confirms the McCain finding of PRE with a small number of acquisition trials. The present data suggest that this effect is not necessarily contradictory to the theoretical position of either Amsel (1958) or Spence (1960) concerning the PRE. Their theory assumes the conditioning of fractional anticipatory frustration responses $\left(r_{f}-s_{f}\right)$ on nonreinforced trials and the subsequent conditioning of the running response to $s_{f}$ on reinforced trials. McCain (1966) has argued that the Amsel-Spence frustration hypothesis cannot predict a PRE after only a few trials because extensive training is necessary to condition $\mathbf{r}_{\mathbf{f}}-\mathbf{s}_{\mathbf{f}}$. However, the results of the present study indicate that the incentive motivation for animals running to different sized rewards is established very early in training and, further, that an emotional response is evident on nonreinforced trials almost immediately. These effects can clearly be seen in Fig. 1 by the large performance increments following rewarded Trials 2 and 4, and the decrement following nonrewarded Trial 3. Accordingly, although fractional anticipatory responses are not fully conditioned within one or two trials, they may be present in sufficient strength to mediate a PRE.

\section{References}

AMSEL, A. The role of frustrative nonreward in noncontinuous reward situations. Psychol Bulh, 1958, 55, 102-119.

ANDERSON, N. H. Comparison of different populations: resistance to extinction and transfer. Psychol. Rev., 1963, 70, 162-179.

LOGAN, F. A. Incentive New Haven: Yale University Press, 1960.

McCAIN, G. Partial reinforcement with a small number of trials: performance in extinction. Psychon. Sci, 1965a, 2, 131-132.

McCAIN, G. Partial reinforcement with a small number of acquisition trials: pretraining and acquisition. Psychon. Sci, 1965b, 2, 249-250.

McCAIN, G. Partial reinforcement effects following a small number of acquisition trials. Psychon. Monogr. Suppl, 1966, 1, 251-270.

SPENCE, K. Behavior theory and learning. Englewood Cliffs: PrenticeHall, Inc., 1960.

Note

1. This research was supported by grant GB-5513X from National Science Foundation to Frank A. Logan. The author gratefully acknowledges Professor Logan for his helpful suggestions and critical reading of the manuscript. 